\title{
Relationship between Traffic Related Air Pollutants and Cognitive Function among Elderly in Egypt
}

\author{
Tomader Taha Abdel Rahman', Maram Maher Monier ${ }^{1 *}$, Sally Adel Hakim², \\ Lamyaa Kamel Ebrahim ${ }^{1}$ \\ ${ }^{1}$ Department of Geriatrics \& Gerontology, Faculty of Medicine, Ain Shams University, Cairo, Egypt \\ ${ }^{2}$ Department of Industrial Medicine, Faculty of Medicine, Ain Shams University, Cairo, Egypt \\ Email: *maram0207@yahoo.com
}

How to cite this paper: Rahman, T.T.A., Monier, M.M., Hakim, S.A. and Ebrahim, L.K. (2019) Relationship between Traffic Related Air Pollutants and Cognitive Function among Elderly in Egypt. Advances in Aging Research, 8, 1-13.

https://doi.org/10.4236/aar.2019.81001

Received: October 30, 2018

Accepted: December 25, 2018

Published: December 28, 2018

Copyright (c) 2019 by authors and Scientific Research Publishing Inc. This work is licensed under the Creative Commons Attribution International License (CC BY 4.0).

http://creativecommons.org/licenses/by/4.0/

\begin{abstract}
Background: Cognitive impairment is a major health issue particularly with the increasing aging population. There are around 47.5 million dementia cases globally. Traffic air pollution issue is a chief environmental problem principally in the mega cities such as Cairo. Methodology: In a Cross sectional and comparative research study the study subjects recruited involved 200 individuals, categorized into two research groups: 100 from Cairo's elderly home residents and 100 from EL-Gharbaya's elderly home residents. Results: Statistical linear regression analysis revealed that fine particulate matter, carbon monoxide, and nitric oxide have a statistically significant impact on cognitive function ( $\mathrm{p}$ values $<0.001$ ) (shown in Table 4). Conclusions: Traffic related air pollutants were strongly associated with cognitive impairment within elderly population in geriatric home residents in Egypt. Regarding to statistically significant difference in concentration of traffic related air pollutants between urban and rural areas, urban areas were more polluted than rural areas.
\end{abstract}

\section{Keywords}

Cognitive Function, Traffic Related Air Pollutants, Elderly in Egypt

\section{Introduction}

Air pollution is a chief issue in public health since various research studies have displayed its several unfavorable health sequalae (e.g. respiratory and cardiovascular diseases). Air pollution has also been considered a prominent environmental risk factor for neurological diseases and neuropathology, and additional- 
ly it is considered to be neurotoxicant [1] [2] [3] [4] [5].

The brain regions correlated to executive functions e.g. working memory and attention are largely presented within the prefrontal cortex and the striatum, which have revealed inflammatory responses after traffic-related air pollution exposure. [6] [7] [8] [9] [10].

Air pollution could also affect the nervous system, in a manner causing memory disturbances, fatigue and hazy vision. Long term exposure to ambient pollution could cause growth of white matter pathological lesions and inhibition of cognitive functions [11] [12] [13] [14] [15].

Ambient air pollution has been correlated to impairment of lung functional performance and chronic obstructive pulmonary disease in the elderly individuals. Impairment of lung function has been correlated with impaired cognitive function. Consequently, cognitive functional decline is highly prevalent in individuals with lung function impairment [16] [17] [18] [19] [20].

Various research studies explored the linkage between midlife pulmonary functional levels and cognitive functional performance in old age which have revealed that pulmonary dysfunction in midlife is correlated with raised risk of poor cognition in elderly life [21] [22] [23] [24] [25].

The influence of pulmonary function on cognitive decline could result from various risk factors involving hypoxia, decreased neurotransmitter function, raised systemic inflammatory processes, or a conjunction of these risk factors [26] [27] [28] [29] [30].

There is rising research based epidemiological evidence of a correlation between air pollution exposure and cognitive functional performance decline, particularly impairment of visuo-construction performance. Epidemiological research studies interestingly reveal and display a correlation between distance to major roads and reduced functions cognitive denoting that particles from traffic pollution could, have detrimental effects on cognition due to either their size or composition [31] [32] [33] [34].

\section{Aim of the Work}

Assessment of the correlation between traffic-related air pollutants exposure and cognitive functional levels is in elderly at geriatric homes in Egypt. Comparison between cognitive function within elderly is exposed to high and low air pollutant levels.

\section{Methodology}

A Cross sectional and comparative research study the study subjects recruited involved 200 individuals, categorized into two research groups: 100 from Cairo's elderly home residents and 100 from EL-Gharbaya's elderly home residents. Inclusive research criteria: Elderly males \& females above 60 years old, accepting to participate in the study, Elderly admitted to geriatric home must come from same area i.e. (in urban nursing homes; their elderly must have a past history of 
living in urban areas) and (in rural nursing homes must had past history of living in rural areas) or at least admitted for 5 years, elderly admitted to those geriatrics homes were apparently healthy. Exclusive research criteria: Refusal to participate in the research study, elderly who has previous history of stroke Oral consent was obtained from 200 study subjects from elderly living in geriatric homes exposed to high \& low traffic related air pollution (urban \& rural areas).

\subsection{Direct Measurements of Traffic Related Geriatric Homes Air Pollution}

Our research team focused on fine particulate matter (PM10), nitric oxide (NO), carbon monoxide (CO) and lead, which are considered to be the most dangerous forms of air pollution. The pollutants measured were real time concentrations. We measured both in Cairo \& in EL-Gharbia related to geriatric home that we recruited the study subjects from them. Measurements were performed by cooperation from Environmental Monitoring Center by usage of a portable field device for measurement of inhaled particles (PM10), lead, carbon monoxide, nitric oxide.

\subsection{Cognitive Assessment}

Cognition was assessed using Cambridge cognition Examination (CAMCOG), by usage of its Arabic version. The CAMCOG is the cognitive and self-contained part of the Cambridge Examination for Mental Disorders of the Elderly (CAMDEX). A standardized instrument for the diagnosis and grading of dementia and assess the level of cognitive impairment. The CAMCOG consists of 67 items with a maximum possible score of 107, and it could be categorized into various subscales: orientation, expressive and comprehensive language, memory (remote, recent, and learning), attention, praxis, calculation, abstraction, and perception. All items of the MMSE are also incorporated into the CAMCOG. If study subject took a score from 90 - 107 there is no cognitive impairment, from 89 - 78 the study subject has mild cognitive impairment, 77 - 66 study subject has mild dementia

Comprehensive clinical geriatric assessment via thorough medical history particularly, Chronic diseases (e.g. hypertension, DM, heart failure, chronic obstructive lung disease) Geriatric depression scale (GDS) using the Arabic translation, Activities of daily living scale (ADL), Instrumental Activities of daily living scale (IADL), Mini Nutritional Assessment (MNA) The sum of the MNA score distinguishes between elderly individuals having: Adequate nutritional status, $\mathrm{MNA} \geq 24$, protein-calorie malnutrition, $\mathrm{MNA}<17$, at risk of malnutrition, MNA between 17 and 23.5.

\subsection{Statistical Analysis}

All data were collected, tabulated, and loaded on personal computer for statistical analysis. As follows: in which description of quantitative research variables as mean, standard deviation (SD) and range, Description of qualitative research va- 
riables as number and percentage, Chi-square test was used to compare qualitative variables between groups, One way Anova test was used to compare quantitative variables between groups, Independent-t-test to compare between two research groups, correlation linear regression model used to assess the most affected air pollutants on cognition. The statistically significant level measured according to $\mathrm{p}$ value, $\mathrm{p}$ value $>0.05$ : Non-significant, $\mathrm{p}$ value $<0.05$ : Significant, $\mathrm{p}$ value $<0.01$ : Highly significant.

\section{Results}

As regards fine particulate matter statistical significant difference existed between Urban and Rural areas being higher in Urban areas ( $\mathrm{p}$ value $<0.001$ ). As regards lead statistical significant difference existed between Urban and Rural areas being higher in Urban areas ( $\mathrm{p}$ value $<0.001$ ).

Concerning carbon monoxide statistical significant difference existed between Urban and Rural areas being higher in Urban areas ( $\mathrm{p}$ value $<0.001$ ). Finally nitric oxide statistical significant difference existed between Urban and Rural areas being higher in Urban areas ( $\mathrm{p}$ value $<0.001$ ).

As regards cognitive function statistically significant correlation existed between age and different grades of cognitive impairment (mild cognitive impairment, mild dementia, moderate to severe cognitive impairment) $\mathrm{p}$ value $<0.001$.

Concerning cognitive impairment correlation to educational level a statistically significant correlation existed in which it was more frequent in illiterate study subjects and least in study subjects having more than 5 years education ( $p$ value $<0.001$ )

Finally gender and smoking didn't show any statistically significant correlation with grades of cognitive functional impairment ( $\mathrm{p}$ values $=0.183,0.345$, consecutively).

As regards fine particulate matter and CAMOG test items and scoring level statistically significant correlation existed in language, Expression, recent memory, Recall \& memory, abstraction, CAMCOG score ( $\mathrm{p}$ values $=0.018,<0.001$, $0.001,<0.001,0.002,0.009$, consecutively).

As regards lead and CAMOG test items and scoring level statistical significant correlation existed in, language, expression, recent memory, recall and memory, abstraction, CAMCOG score ( $\mathrm{p}$ value $=0.030,<0.001,<0.001,<0.001,0.002$, 0.011 , consecutively).

Concerning carbon monoxide and CAMOG test items and scoring level statistically significant correlation existed in orientation, language, expression, recent memory, remote memory, attention, drawing, recall and memory, abstraction, perception, CAMCOG score ( $\mathrm{p}$ value $=0.022,0.002,<0.001,<0.001,0.028$, $0.032,<0.001,<0.001,0.006,<0.001$, consecutively).

Concerning nitric oxide and CAMOG test items and scoring level statistically significant correlation existed in Expression, recent memory, praxis recall and memory, abstraction, CAMCOG score $(\mathrm{p}$ value $=0.001,0.002,0.021,0.001$, 
0.006, 0.039, consecutively).

Statistical linear regression analysis revealed that fine particulate matter, carbon monoxide, and nitric oxide have a statistically significant impact on cognitive function ( $\mathrm{p}$ values $<0.001$ ).

As regards Geriatric depression scale scoring and air pollutants components (fine particulate matter, lead carbon monoxide, nitric oxide) statistical significant correlation existed in all items ( $\mathrm{p}$ values $=0.002,0.001,0.001,0.004$, consecutively), instrumental activities of daily living scale scoring and air pollutants components (statistical significant correlation existed in fine particulate matter, lead carbon monoxide, $\mathrm{p}$ values $=0.041,0.046,0.005$, consecutively), regarding activities of daily living scale scoring and air pollutants components no statistical significant correlations existed.

\section{Discussion}

Cognitive impairment is a major health issue particularly with the increasing aging population. There are around 47.5 million dementia cases globally, newly diagnosed cases every year is about 7.7 million case. Dementia was ranked first among chronic non-communicable diseases in low and middle income nations. Within the next four decades, the prevalence of dementia is estimated to triple except protective measures are implemented. With increased levels of urbanization and road traffic expansion in various areas of the world, population exposed to air pollution is raised particularly in older adults [1] [3] [6] [7].

Traffic air pollution issue is a chief environmental problem principally in the mega cities such as Cairo. It was considered as the 12th leading risk factor for disability in 2013 globally, causing about 3.3 million premature deaths each year [10] [13] [15].

The current research study was conducted to evaluate the correlation between cognitive impairment and traffic air pollution within elderly home residents in urban and rural areas.

The study recruited 200 elderly study subjects (60 years old and above), both males and females have been recruited from elderly home residents (100 from Cairo's and 100 from EL-Gharbia's elderly home residents).

In the current research study the research team the following results were obtained concerning fine particulate matter(shown in Table 1) statistical significant difference existed between Urban and Rural areas being higher in Urban areas ( $\mathrm{p}$ value $<0.001$ ). As regards lead statistical significant difference existed between Urban and Rural areas being higher in Urban areas ( $p$ value $<0.001$ ). As regards carbon monoxide statistical significant difference existed between Urban and Rural areas being higher in Urban areas ( $p$ value $<0.001$ ). Finally nitric oxide statistical significant difference existed between Urban and Rural areas being higher in Urban areas ( $\mathrm{p}$ value $<0.001$ ).

The mean concentration levels of air pollutants in urban areas exceeded the standard environmental levels, especially fine particulate matter, in accordance to Environmental monitoring center report in Egypt. These findings are in harmony 
Table 1. Differences between air pollutants level in urban and rural areas.

\begin{tabular}{|c|c|c|c|c|c|c|c|c|c|}
\hline \multirow{2}{*}{\multicolumn{2}{|c|}{ Air pollutants }} & \multicolumn{6}{|c|}{ Area } & \multicolumn{2}{|c|}{ T-Test } \\
\hline & & \multicolumn{3}{|c|}{ Urban } & \multicolumn{3}{|c|}{ Rural } & $\mathrm{T}$ & p-value \\
\hline \multirow{2}{*}{ PM10 } & Range & 269 & - & 275 & 202 & - & 212 & \multirow{2}{*}{166.687} & \multirow{2}{*}{$<0.001^{\star}$} \\
\hline & Mean \pm SD & 270.690 & \pm & 2.343 & 207.430 & \pm & 2.986 & & \\
\hline \multirow{2}{*}{ Lead } & Range & 0.20 & - & 0.24 & 0.02 & - & 0.03 & \multirow{2}{*}{68.368} & \multirow{2}{*}{$<0.001^{\star}$} \\
\hline & Mean \pm SD & 0.209 & \pm & 0.027 & 0.024 & \pm & 0.005 & & \\
\hline \multirow{2}{*}{ Co } & Range & 16 & - & 19 & 6 & - & 10 & \multirow{2}{*}{47.189} & \multirow{2}{*}{$<0.001^{\star}$} \\
\hline & Mean $\pm S D$ & 17.320 & \pm & 1.497 & 8.380 & \pm & 1.162 & & \\
\hline \multirow{2}{*}{ No } & Range & 4.9 & - & 5.1 & 0 & - & 0.2 & \multirow{2}{*}{375.824} & \multirow[b]{2}{*}{$<0.001^{\star}$} \\
\hline & Mean \pm SD & 5.001 & \pm & 0.100 & 0.048 & \pm & 0.086 & & \\
\hline
\end{tabular}

$\mathrm{p}$ value $>0.05$ : Non-significant; $\mathrm{p}$ value $<0.05$ : Significant; $\mathrm{p}$ value $<0.01$ : Highly significant.

with various research studies including a research study previously conducted by Apăscăriței et al. research team of investigators in which they revealed that NO levels were ten times higher in urban than in rural area also CO levels were two times higher in urban area [18] [25] [27].

As regards the correlation between age, sex, educational level, smoking and cognition in all study cohort of the current research (revealed and displayed in Table 2) the following was revealed in which cognitive function there was a statistically significant correlation that existed between age and different grades of cognitive impairment (mild cognitive impairment, mild dementia, moderate to severe cognitive impairment) $\mathrm{p}$ values $<0.001$.

Concerning cognitive impairment correlation to educational level a statistically significant correlation existed (shown in Table 2) in which it was more frequently observed in illiterate study subjects and least in study subjects having more than 5 years education ( $\mathrm{p}$ value $<0.001)$. Finally gender and smoking didn't show any statistical significant correlation with grades of cognitive functional impairment. ( $\mathrm{p}$ values $=0.183,0.345$, consecutively)

These results and observations obtained are consistent with a research study performed by Ji et al., research team of investigators who revealed and displayed from their research data that the prevalence of cognitive impairment and dementia was $73.2 \%$ within the elderly individuals aged 80 years and older and the prevalence of dementia and Alzheimer's disease within elderly individuals aged 60 years and older have been $7.7 \%$. these findings were in harmony with the current research study. On the other hand Ji et al., research team revealed that the prevalence of cognitive impairment was 2.3 -fold higher in females than in males and was raised by 1.8 -fold in females than in males aged 80 years old and older, that contradicts with the current research in which no gender differences were observed among the cohort as regards cognitive impairment [2] [5] [12] [22].

These findings are explained by the fact that the aging process is accompanied 
Table 2. Relation between age, sex, educational level, smoking and cognition in all study population.

\begin{tabular}{|c|c|c|c|c|c|c|c|c|c|c|c|c|c|c|c|}
\hline & & \multicolumn{12}{|c|}{ Total score } & \multicolumn{2}{|c|}{$\begin{array}{l}\text { ANOVA or } \\
\text { Chi-Square }\end{array}$} \\
\hline & & \multicolumn{3}{|c|}{ Normal } & \multicolumn{3}{|c|}{ MCI } & \multicolumn{3}{|c|}{ Mild dementia } & \multicolumn{3}{|c|}{ Moderate to severe } & F or $X^{2}$ & p-value \\
\hline \multirow[b]{2}{*}{ Age } & Range & 60 & - & 84 & 60 & - & 83 & 62 & - & 96 & 60 & - & 91 & \multirow[b]{2}{*}{14.212} & \multirow[b]{2}{*}{$<0.001^{*}$} \\
\hline & Mean \pm SD & 69.515 & \pm & 5.719 & 71.750 & \pm & 6.142 & 77.231 & \pm & 7.773 & 76.895 & \pm & 9.116 & & \\
\hline \multirow{2}{*}{ Sex } & Male & 53 & & 51.46 & 25 & & 48.08 & 10 & & 38.46 & 5 & & 26.32 & \multirow{2}{*}{4.856} & \multirow{2}{*}{0.183} \\
\hline & Female & 50 & & 48.54 & 27 & & 51.92 & 16 & & 61.54 & 14 & & 73.68 & & \\
\hline \multirow{3}{*}{ Education } & Illiterate & 2 & & 1.94 & 12 & & 23.08 & 13 & & 50.00 & 13 & & 68.42 & \multirow{3}{*}{78.389} & \multirow{3}{*}{$<0.001^{*}$} \\
\hline & Less than 5 years & 13 & & 12.62 & 11 & & 21.15 & 6 & & 23.08 & 5 & & 26.32 & & \\
\hline & More than 5 years & 88 & & 85.44 & 29 & & 55.77 & 7 & & 26.92 & 1 & & 5.26 & & \\
\hline Smoking & Smoker & 33 & & 32.04 & 22 & & 42.31 & 8 & & 30.77 & 4 & & 21.05 & 3.318 & 0.345 \\
\hline
\end{tabular}

by influences in the leukocytes functional performance and the antioxidant defense system that could predispose elderly individuals to inappropriate amount and harmful inflammatory pathological responses that have negative impact on cognitive function [15] [32] [34].

However, another research study by Bai et al., research team of investigators have revealed that cognitive levels of affection have not been to gender and that finding was similarly revealed by the current research study [30] [33].

As regards cognitive impairment correlation to educational level in the current research study a statistically significant correlation existed in which it was more frequent in illiterate study subjects and least in study subjects having more than 5 years education ( $\mathrm{p}$ value $<0.001$ ).

These findings are in harmony with a prior research study that revealed that the risk of cognitive impairment within elderly individuals with education levels of less than 6 years in comparison to those elderly individuals with education levels of 9 years or more, with a 3.8 -fold increase in the illiterate research group and a 1.7-fold rise in those with 1 - 5 years of education.

Another research study conducted by Elwan et al. research team showed similar results to the current research as regards level of education in which the Illiterate research group and the group with primary school level of education had a low scoring level concerning short term memory, psychomotor functional performance and perception than control research group that was highly educated. Smoking is a possible risk factor for dementia as it may increase free radicals generation but its impact could be underestimated within older adult due to the shorter life span of smokers [10] [16] [22].

The current research study revealed that there was no correlation between cognitive impairment levels and smoking within the elderly, no statistically significant difference existed between normal elderly and who had cognitive impairment as regard smoking habit.

It was consistent with the study of Hebert et al., research team in East Boston, 
that revealed that there was no correlation was observed between smoking and cognitive decline within elderly. On the other hand, another research study previously performed displayed and revealed that current smoking raises the risk of cognitive decline and developing of dementia, and quitting smoking could reduce the associated risk to levels comparable to those who have never smoked [25] [28] [33].

Concerning the statistical correlation between items of CAMOG test components and air pollutants in study cohort (revealed and displayed in Table 3). As regards fine particulate matter and CAMOG test items and scoring level statistical significant correlation existed in language, Expression, recent memory, Recall \& memory, abstraction, CAMCOG score ( $\mathrm{p}$ values $=0.018,<0.001,0.001$, $<0.001,0.002,0.009$, consecutively) (revealed and displayed in Table 3). As regards lead and CAMOG test items and scoring level statistical significant correlation existed in language, expression, recent memory, recall and memory, abstraction, CAMCOG score ( $\mathrm{p}$ value $=0.030,<0.001,<0.001,<0.001,0.002,0.011$, consecutively). (Revealed and displayed in Table 3). Concerning carbon monoxide and CAMOG test items and scoring level statistical significant correlation existed in orientation, language, expression, recent memory, remote memory, attention, drawing, recall and memory, abstraction, perception, CAMCOG score $(\mathrm{p}$ value $=0.022,0.002,<0.001,<0.001,0.028,0.032,<0.001,<0.001,0.006$, $<0.001$, consecutively). (Revealed and displayed in Table 3 ). Concerning nitric

Table 3. Correlation between item of CAMOG test and air pollutants in all study population.

\begin{tabular}{|c|c|c|c|c|c|c|c|c|}
\hline \multicolumn{9}{|c|}{ Correlations } \\
\hline & \multicolumn{2}{|c|}{ PM10 } & \multicolumn{2}{|c|}{ Lead } & \multicolumn{2}{|c|}{ Co } & \multicolumn{2}{|c|}{ No } \\
\hline & $\mathbf{r}$ & $\mathrm{p}$-value & $\mathbf{R}$ & p-value & $\mathbf{r}$ & p-value & $\mathbf{R}$ & p-value \\
\hline Orientation & -0.081 & 0.254 & -0.102 & 0.152 & -0.161 & $0.022^{\star}$ & -0.048 & 0.500 \\
\hline Language & -0.167 & $0.018^{\star}$ & -0.153 & $0.030^{\star}$ & -0.214 & $0.002^{\star}$ & -0.133 & 0.060 \\
\hline Expression & -0.272 & $<0.001^{*}$ & -0.257 & $<0.001^{*}$ & -0.347 & $<0.001^{\star}$ & -0.242 & $0.001^{*}$ \\
\hline Recent memory & -0.241 & $0.001^{*}$ & -0.245 & $<0.001^{*}$ & -0.301 & $<0.001^{*}$ & -0.214 & $0.002^{*}$ \\
\hline Remote memory & -0.080 & 0.260 & -0.068 & 0.336 & -0.155 & $0.028^{*}$ & -0.047 & 0.504 \\
\hline Registration & 0.105 & 0.137 & 0.102 & 0.151 & 0.056 & 0.432 & 0.115 & 0.105 \\
\hline Attention & -0.103 & 0.147 & -0.118 & 0.097 & -0.151 & $0.032^{\star}$ & -0.070 & 0.324 \\
\hline Drawing & -0.103 & 0.145 & -0.086 & 0.224 & -0.163 & $0.021^{\star}$ & -0.069 & 0.335 \\
\hline Praxis & 0.127 & 0.072 & 088.126 & 0.075 & 0.067 & 0.349 & 0.163 & $0.021^{*}$ \\
\hline Calculation & 0.107 & 0.130 & 0.102 & 0.153 & 0.076 & 0.285 & 0.109 & 0.123 \\
\hline Recall \& memory & -0.268 & $<0.001^{*}$ & -0.252 & $<0.001^{*}$ & -0.327 & $<0.001^{*}$ & -0.239 & $0.001^{*}$ \\
\hline Abstraction & -0.217 & $0.002^{*}$ & -0.221 & $0.002^{*}$ & -0.290 & $<0.001^{*}$ & -0.195 & $0.006^{*}$ \\
\hline Perception & -0.097 & 0.170 & -0.111 & 0.116 & -0.195 & $0.006^{\star}$ & -0.072 & 0.3127 \\
\hline CAMCOG score & -0.184 & $0.009^{*}$ & -0.179 & $0.011^{*}$ & -0.256 & $<0.001^{*}$ & -0.146 & $0.039^{*}$ \\
\hline
\end{tabular}


oxide and CAMOG test items and scoring level statistically significant correlation existed in Expression, recent memory, praxis recall and memory, abstraction, CAMCOG score ( $\mathrm{p}$ value $=0.001,0.002,0.021,0.001,0.006,0.039$, consecutively) (Revealed and displayed in Table 3). Therefore, in general air pollutants had a statistically significant impact on the scoring of CAMCOG test in both urban and rural research study cohort but its impact in urban research group was more than on rural research that revealed high cognitive impairment level than in rural area (Table $4 \&$ Table 5).

Contradicting to the current research study findings, a prior research performed which evaluated the cognitive impairment prevalence in the Portuguese population in both urban and rural areas, revealed and displayed that cognitive impairment levels within rural areas were higher than that in urban areas. This discrepancy between could be explained by difference in education levels and age not due to air pollution differences [14] [18] [20].

A study applied on urban population within Columbia revealed that air pollution have harmful impact on health of elderly even when the level of pollutants is low, this finding contradicts with the current research results. However this finding could explained by the influence of other factors such as climate change and temperature [14] [18] [23].

A prospective cohort research study of Wellenius et al., research team which observed an association between $\mathrm{CO}$ and immediate recall in 756 elderly women; however, there were no clear correlations with expression and orientation also

Table 4. Linear regression, and important variables on multivariate analysis were introduced in stepwise linear regression model to detect effect of air pollutants on cognitive function in all study population.

\begin{tabular}{cccccc}
\hline & \multicolumn{2}{c}{ Unstandardized Coefficients } & $\begin{array}{c}\text { Standardized } \\
\text { Coefficients }\end{array}$ & $\mathbf{t}$ & p-value \\
\cline { 2 - 4 } & B & Std. Error & Beta & & \\
\hline PM10 & 0.191 & 0.023 & 6.112 & 8.256 & $<0.001^{*}$ \\
Lead & 1.497 & 2.934 & 0.143 & 0.510 & 0.611 \\
Co & 0.256 & 0.044 & 1.204 & 5.802 & $<0.001^{\star}$ \\
No & -2.913 & 0.289 & -7.267 & -10.097 & $<0.001^{\star}$ \\
\hline
\end{tabular}

Dependent Variable: CAMCOG scores.

Table 5. Correlation between the function (ADL, IADL), depression (GDS) and air pollutants in all study population.

\begin{tabular}{ccccccc}
\hline & \multicolumn{2}{c}{ ADL } & \multicolumn{2}{c}{ IADL } & \multicolumn{2}{c}{ GDS } \\
\cline { 2 - 7 } & r & p-value & r & p-value & r & p-value \\
\hline PM10 & 0.006 & 0.936 & -0.145 & $0.041^{*}$ & 0.221 & $0.002^{*}$ \\
Lead & -0.004 & 0.956 & -0.141 & $0.046^{*}$ & 0.225 & $0.001^{*}$ \\
Co & -0.066 & 0.353 & -0.196 & $0.005^{*}$ & 0.223 & $0.001^{*}$ \\
No & 0.034 & 0.630 & -0.117 & 0.099 & 0.203 & $0.004^{*}$ \\
\hline
\end{tabular}


found that closer proximity of residence to major roads was associated with poorer performance on several cognitive tests, especially that assess executive function, memory, verbal learning and language [30] [33].

Another research study evaluating impact of long-term exposure to fine particulate matter cognition decline levels in elderly women in the United States found that a $10 \mu \mathrm{g} / \mathrm{m}^{3}$ increase in fine particulate matter has been linked to a 0.014 fall in verbal memory; 0.002 decrease in verbal fluency. A study of Schikowski et al., research team in harmony with the current research study results revealed that nitric oxide levels are correlated to a decrease in visuospatial abilities, naming and memory [10] [26].

\section{Conclusion}

The current study showed that traffic related air pollutants were strongly associated with cognitive impairment within elderly population in geriatric home residents in Egypt. Regarding to statistically significant difference in concentration of traffic related air pollutants between urban and rural areas, urban areas were more polluted than rural areas. Air pollutants have a negative impact on recall, memory, expression and language, and minimal impact on attention, drawing, and perception and had no clear impact on orientation calculation, and registration.

\section{Recommendations}

Screening for cognitive impairment of all elderly persons attending health centers should be carried out. Continuation physical, mental, social activities and early detection and management of depression is required to reduce cognitive impairment in elderly. Improvements to air quality may be an important mechanism for reducing cognitive decline in elderly. Large scale research studies both observational and interventional are required for additional evaluation of air pollution impact on elderly within Egypt. Future research studies should be multicentric in fashion and put in consideration racial and ethnic differences.

\section{Conflicts of Interest}

The authors declare no conflicts of interest regarding the publication of this paper.

\section{References}

[1] Grandjean, P. and Landrigan, P.J. (2014) Neurobehavioral Effects of Developmental Toxicity. The Lancet Neurology, 13, 330-338. https://doi.org/10.1016/S1474-4422(13)70278-3

[2] Gerlofs-Nijland, M.E., van Berlo, D., Cassee, F.R., et al. (2010) Effect of Prolonged Exposure to Diesel Engine Exhaust on Proinflammatory Markers in Different Regions of the Rat Brain. Particle and Fibre Toxicology, 7, 12. 
[3] Power, M.C., Weisskopf, M.G., Alexeeff, S.E., et al. (2011) Traffic-Related Air Pollution and Cognitive Function in a Cohort of Older Men. Environmental Health Perspectives, 119, 682-627.

[4] Bennett, I.J. and Madden, D.J. (2014) Disconnected Aging: Cerebral White Matter Integrity and Age-Related Differences in Cognition. Neuroscience, 276, 187-205. https://doi.org/10.1016/j.neuroscience.2013.11.026

[5] Jekel, K., Damian, M., Wattmo, C., et al. (2015) Mild Cognitive Impairment and Deficits in Instrumental Activities of Daily Living: A Systematic Review. Alzheimer's Research \& Therapy, 7, 17. https://doi.org/10.1186/s13195-015-0099-0

[6] Harada, C.N., Love, M.C. and Triebel, K.L. (2013). Normal Cognitive Aging. Clinics in Geriatric Medicine, 29, 737-752. https://doi.org/10.1016/j.cger.2013.07.002

[7] Davis, H.P., Klebe, K.J., Guinther, P.M., et al. (2013) Subjective Organization, Verbal Learning, and Forgetting Across the Life Span: From 5 to 89. Experimental Aging Research, 39, 1-26. https://doi.org/10.1080/0361073X.2013.741956

[8] Rogalski, E., Stebbins, G.T., Barnes, C.A., et al. (2012) Age-Related Changes in Para hippocampal White Matter Integrity: A Diffusion Tensor Imaging Study. Neuropsychologia, 50, 1759-1765. https://doi.org/10.1016/j.neuropsychologia.2012.03.033

[9] Lin, J.S., O’Connor, E., Rossom, R.C., et al. (2013) Screening for Cognitive Impairment in Older Adults: A Systematic Review for the US Preventive Services Task Force. Annals of Internal Medicine, 159, 601-612. https://doi.org/10.7326/0003-4819-159-9-201311050-00730

[10] Wisdom, N.M., Mignogna, J. and Collins, R.L. (2012) Variability in Wechsler Adult Intelligence Scale-IV Subtest Performance across Age. Archives of Clinical Neuropsychology, 27, 389-397. https://doi.org/10.1093/arclin/acs041

[11] World Health Organization (2011) Urban Outdoor Air Pollution Database. Geneva.

[12] Ramadan, I.M. and Rashwan, N.K. (2015) Calibration of Vehicle Emissions-Speed Relationships for the Greater Cairo Roads. International Journal of Civil Engineering and Technology, 7, 1.

[13] Shandilya, K.K. and Khare, M. (2012) Particulate Matter: Sources, Emission Rates and Health Effects. Advances in Environmental Research, 23, 265-322.

[14] Moawad, M.B., Youssief, A.A. and Madkour, K. (2017) Modeling and Monitoring of Air Quality in Greater Cairo Region, Egypt Using Landsat-8 Images, HYSPLIT and GIS Based Analysis. Climate Change Research at Universities, Springer International Publishing, 37-54, Berlin.

[15] Robaa, S.M. (2013) Some Aspects of the Urban Climates of Greater Cairo Region, Egypt. International Journal of Climatology, 33, 3206-3216. https://doi.org/10.1002/joc.3661

[16] Kim, K.H., Jahan, S.A., Kabir, E. et al. (2013) A Review on Human Health Perspective of Air Pollution with Respect to Allergies and Asthma. Environment International, 59, 41-52. https://doi.org/10.1016/j.envint.2013.05.007

[17] Shah, A.S., Langrish, J.P., Nair, H., et al. (2013) Global Association of Air Pollution and Heart Failure: A Systematic Review and Meta-Analysis. The Lancet, 382, 1039-1048. https://doi.org/10.1016/S0140-6736(13)60898-3

[18] Burnett, R.T., Pope III, C.A., Ezzati, M., et al. (2014) An Integrated Risk Function for Estimating the Global Burden of Disease Attributable to Ambient Fine Particulate Matter Exposure. Environmental Health Perspectives, 122, 397.

[19] Lelieveld, J., Evans, J.S., Fnaiset, M., et al. (2015) The Contribution of Outdoor Air 
Pollution Sources to Premature Mortality on a Global Scale. Nature, 525, 367-371. https://doi.org/10.1038/nature15371

[20] Kelly, F.J. and Fussell, J.C. (2012) Size, Source and Chemical Composition as Determinants of Toxicity Attributable to Ambient Particulate Matter. Atmospheric Environment, 60, 504-526. https://doi.org/10.1016/j.atmosenv.2012.06.039

[21] Zhang, J.J. and Samet, J.M. (2015) Chinese Haze versus Western Smog: Lessons Learned. Journal of Thoracic Disease, 7, 3-13.

[22] Cadelis, G., Tourres, R. and Molinie, J. (2014) Short-Term Effects of the Particulate Pollutants Contained in Saharan Dust on the Visits of Children to the Emergency Department Due to Asthmatic Conditions in Guadeloupe (French Archipelago of the Caribbean). PLOS ONE, 9, e91136. https://doi.org/10.1371/journal.pone.0091136

[23] Esworthy, R. (2013) Air Quality: EPA's 2013 Changes to the Particulate Matter (PM) Standard. Congressional Research Service, 7-5700.

[24] Perez, L., Tobías, A., et al. (2012) Saharan Dust, Particulate Matter and Cause-Specific Mortality: A Case-Crossover Study in Barcelona (Spain). Environment International, 48, 150-155. https://doi.org/10.1016/j.envint.2012.07.001

[25] Calderón-Garcidueñas, L., Leray, H.P., et al. (2016) Air Pollution, a Rising Environmental Risk Factor for Cognition, Neuroinflammation and Neurodegeneration: The Clinical Impact on Children and Beyond. Revue Neurologique, 172, 69-80. https://doi.org/10.1016/j.neurol.2015.10.008

[26] Wohleb, E.S., McKim, Sheridan, J.F., et al. (2015) Monocyte Trafficking to the Brain with Stress and Inflammation: A Novel Axis of Immune-to-Brain Communication That Influences Mood and Behavior. Frontiers in Neuroscience, 8, 447. https://doi.org/10.3389/fnins.2014.00447

[27] Rodrigues, R., Petersen, R.B. and Perry, G. (2014) Parallels between Major Depressive Disorder and Alzheimer's Disease: Role of Oxidative Stress and Genetic Vulnerability. Cellular and Molecular Neurobiology, 34, 925-949. https://doi.org/10.1007/s10571-014-0074-5

[28] Heusinkveld, H.J., Wahle, T., Campbell, A., et al. (2016) Neurodegenerative and Neurological Disorders by Small Inhaled Particles. Neurotoxicology, 56, 94-106. https://doi.org/10.1016/j.neuro.2016.07.007

[29] Steenhof, M., Janssen, N.A., Strak, M., et al. (2014) Air Pollution Exposure Affects Circulating White Blood Cell Counts in Healthy Subjects: The Role of Particle Composition, Oxidative Potential and Gaseous Pollutants-The RAPTES Project. Inhalation Toxicology, 26, 141-165. https://doi.org/10.3109/08958378.2013.861884

[30] Chiu, I.M., Von Hehn, C.A. and Woolf, C.J. (2012) Neurogenic Inflammation and the Peripheral Nervous System in Host Defense and Immunopathology. Nature Neuroscience, 15, 1063. https://doi.org/10.1038/nn.3144

[31] Mumaw, C.L., Levesque, S., McGraw, C., et al. (2016) Microglial Priming through the Lung-Brain Axis: The Role of Air Pollution-Induced Circulating Factors. The FASEB Journal, 30, 1880-1891. https://doi.org/10.1096/fj.201500047

[32] Ji, Y., Shi, Z., Zhang, Y., et al. (2015) Prevalence of Dementia and Main Subtypes in Rural Northern China. Dementia and Geriatric Cognitive Disorders, 39, 294-302. https://doi.org/10.1159/000375366

[33] Ren, L., Bai, L., Wu, Y., et al. (2018) Prevalence of and Risk Factors for Cognitive Impairment among Elderly without Cardio- and Cerebrovascular Diseases: A Population-Based Study in Rural China. Frontiers in Aging Neuroscience, 10, 62. 
https://doi.org/10.3389/fnagi.2018.00062

[34] Clifford, A., Lang, L., Chen, R., et al. (2016) Exposure to Air Pollution and Cognitive Functioning across the Life Course-A Systematic Literature Review. Environmental Research, 147, 383-398. https://doi.org/10.1016/j.envres.2016.01.018 